\title{
ILCEA
}

Revue de l'Institut des langues et cultures

d'Europe, Amérique, Afrique, Asie et Australie

33 | 2018

Femmes en résistance du XVIIIe siècle à nos jours

\section{Les résistances féminines de réassurance face au dépouillement matériel et à la dépossession éthique du franquisme}

Ante el despojo material y la desposesión ética del Franquismo, resistencias

femeninas de reaseguración

In the Face of Francoist Material Plundering and Ethical Dispossession, Feminine

Resistance and Empowerment

Irene Murillo Aced

\section{CpenEdition}

Journals

Édition électronique

URL : http://journals.openedition.org/ilcea/5144

DOI : 10.4000/ilcea.5144

ISSN : 2101-0609

Éditeur

UGA Éditions/Université Grenoble Alpes

Édition imprimée

ISBN : 978-2-37747-060-0

ISSN : 1639-6073

Référence électronique

Irene Murillo Aced, « Les résistances féminines de réassurance face au dépouillement matériel et à la dépossession éthique du franquisme », ILCEA [En ligne], 33 | 2018, mis en ligne le 05 novembre 2018, consulté le 01 mai 2019. URL : http://journals.openedition.org/ilcea/5144 ; DOI : 10.4000/ilcea.5144

Ce document a été généré automatiquement le 1 mai 2019.

(c) ILCEA 


\title{
Les résistances féminines de
} réassurance face au dépouillement matériel et à la dépossession éthique du franquisme ${ }^{1}$

\author{
Ante el despojo material y la desposesión ética del Franquismo, resistencias \\ femeninas de reaseguración \\ In the Face of Francoist Material Plundering and Ethical Dispossession, Feminine \\ Resistance and Empowerment
}

Irene Murillo Aced

1 La Communauté de la victoire lancée par l'État franquiste orchestra toutes sortes de mesures légales, sociales, physiques, sexuelles, économiques, symboliques, politiques et professionnelles pour l'annulation et la dépossession de sujets qui étaient associés à l'«Autre» républicain. Un vaste système fut créé à ces fins, notamment avec la promulgation de la Loi sur les responsabilités politiques en 1939 (ci-après dénommée LRP). Celle-ci constitua l'un des outils législatifs utilisés par le régime de Franco pour légitimer le coup d'État, consolider sa victoire par les armes et obtenir le contrôle sur la population durant l'après-guerre (Del Arco Blanco, 2009 ; voir aussi Anderson, 2013 ; Aró stegui, 2012; Tamarit Sumalla, 2005). Le texte même de la loi ne laissait aucun doute quant à son objectif :

[...] sanctionner les fautes politiques de ceux qui, par leurs actes ou leurs omissions graves, ont contribué à forger la subversion rouge, à l'entretenir pendant plus de deux ans et à entraver la victoire providentielle et historiquement inéluctable du Mouvement national'2.

2 Ses mécanismes: les amendes, les interdictions professionnelles et les déportations. Lorsque ces sanctions ne pouvaient pas être exécutées, elles se transformaient en saisies, expulsions en droit et ventes de biens aux enchères publiques. 
3 La LRP n'est pas une simple loi, mais le paradigme même de cette double volonté ré pressive : la mort civile et sociale de l'« ennemi » par la dépossession matérielle de ses biens et moyens de subsistance et par le dépouillement immatériel lié à la volonté du régime de possession des corps, soumis à une violence militaire, professionnelle, sexuelle et économique. Pour ce qui est de l'aspect matériel, l'objectif était de punir et d'exposer à l'humiliation publique, par l'expropriation, l'interdiction et la sanction économique, ceux qui avaient été rattachés au régime républicain ou à la lutte antifasciste. Dans la région d'Aragon, où des sanctions furent appliquées à plus de trois mille personnes, ces républicains et républicaines auraient selon la dictature «porté préjudice à un corps politique national inexistant, qui n'en était encore qu'à ses balbutiements et ne serait ratifié que postérieurement, par sa victoire par les armes, à hauteur de plus de 20 millions de pesetas» (Langarita, Moreno \& Murillo, 2014). Sur le plan macroéconomique, ce régime de possession, qui limitait la propriété à un nombre de personnes très restreint, laissait le reste de la population démuni et condamné à la pauvreté. Les lois classistes menées par les politiques économiques du régime gelèrent le statu quo entre les riches et les pauvres, creusant davantage la brèche séparant les premiers, qui bénéficièrent des conséquences de la guerre, des seconds, qui subissaient toute la misère des années d'autarcie. Néanmoins, il est également important d'aborder le cas des femmes espagnoles d'après-guerre, à qui nous dédierons cet article. En effet, l'adoption de lois de portée clairement anti-féministe visait à la mise en place de politiques de maternité forcée, qui contribuèrent à ancrer les mécanismes de soumission des femmes aux hommes (Nash, 1996 ; voir aussi Cenarro, 2016 ; Molinero, 1998 ; Valiente, 2003).

D'un point de vue moins matériel, le franquisme aspirait à déposséder la population des expériences démocratiques qui lui conféraient une liberté relative dans la sphère publique, ainsi que des premiers changements relatifs à la sphère privée, qui avaient permis aux femmes d'acquérir certaines formes de respect. De même, la volonté de détruire les relations personnelles, de voisinage ou d'amitié prit davantage d'ampleur, en cherchant à rompre jusqu'aux liens d'attention, de collaboration, de soutien, de soin et de solidarité primaires qui contribuaient à façonner la conception de l'autre comme un égal. Par ailleurs, par la privatisation de la souffrance sociale découlant de la guerre civile comme phénomène exclusif du camp vainqueur, il fut interdit aux victimes d'évacuer leur peine et de raconter leurs souvenirs. Nous dédierons cet article à ce sujet, qui suscita dès le départ des résistances.

Dans cet environnement créé par l'adoption de la LRP, en plus des femmes directement sanctionnées pour leurs actions, certaines femmes furent également co-responsabilisées, forcées de répondre, en tant qu'épouses et mères, des actions des hommes de leur famille. Ainsi, les femmes subirent à la fois une répression directe, en tant que responsables politiques, et indirecte, en tant que conjointes de responsables politiques (Mir, 1997, 2004 ; voir aussi Barrado, 1999). À cet égard, la documentation fait état de centaines de femmes qui se défendirent contre les saisies, cherchèrent à éviter le paiement des amendes et usèrent de tous les délais et voies administratives possibles pour empêcher 1 'application de la sanction, tentant de minimiser ainsi les effets de la Loi au quotidien. Ces pratiques et stratégies pourraient selon nous être qualifiées, sans nulle peur de l'ambiguï té, de résistances quotidiennes au franquisme. Ces résistances supposeraient la recherche d'une réassurance matérielle et immatérielle, comme nous pourrons le voir au fil de cet article. 
6 Nous consacrerons une partie de cet article à décrire les moyens utilisés par ces femmes, principalement des veuves de républicains, qui refusaient d'accepter la version officielle de la guerre et de la violence, ainsi que la façon dont la législation était appliquée et dont les biens étaient saisis. Face à une mémoire usurière considérant que l'organe endommagé suite au conflit était l'organe politique de la nation et que le collectif républicain était l'auteur matériel de tous les dommages qu'avaient subis la nation et les individus, se dressaient les contestations de ceux qui avaient connu l'autre version de cette hégémonie du souvenir. Face à la dépréciation des expériences individuelles et à l'expulsion de la douleur du domaine public, les récits de ces personnes n'étaient pas seulement émotionnels et domestiques, bien que basés sur leurs propres expériences et sentiments, mais ils intégraient également, par le biais de l'émotion, l'arme politique qu'était la connaissance de ce qui s'était produit, le fait de savoir quelque chose que l'État niait.

7 Nous proposerons ci-dessous un bref aperçu de l'historiographie des résistances menées par les femmes, pour ensuite nous concentrer sur un type de résistance spécifique de l'Espagne franquiste.

\section{Un bref aperçu historiographique de la résistance féminine}

Sur le plan historiographique, il est peu utile de rechercher dans les régimes d'absence de libertés et de droits, tels que la dictature franquiste espagnole (1939-1975), des oppositions ouvertes ou des formes de militantisme similaires à celles qui apparurent durant la Seconde République espagnole, un régime démocratique conférant des droits civils à la population. Dans le cadre du franquisme, les oppositions et dissidences prenaient des formes plus subtiles et quotidiennes. Face à une grande variété d'opinions et de comportements relatifs au régime, nous devons tenir compte de différentes variables telles que l'expérience même de la violence (personnelle ou familiale) durant la guerre, les répercussions positives ou négatives des politiques économiques, le rapport au catholicisme, etc. (Del Arco Blanco, Hernández Burgos \& Marco, 2013). Durant l'aprèsguerre espagnole, les résistances féminines au régime franquiste se démarquèrent des oppositions ouvertes et frontales pour transiter vers des terrains davantage propices aux voies détournées, aux négociations et à l'illégalité. Rendre ces expériences invisibles impliquerait, comme l'explique Anna Bravo, d'ériger les fondements de la citoyenneté sur des absences théoriques importantes et une insuffisance dangereuse pour les imaginaires collectifs qui s'abreuvaient uniquement d'expériences masculines, autrement dit de prototypes construits sur l'héroïcité, la politique publique, le syndicalisme, les complots, la violence et la prise de pouvoir (Bravo, 1995).

9 En Espagne, deux courants historiographiques se sont toujours côtoyés dans l'étude des résistances: l'un axé sur les stratégies de survie et l'autre axé sur les résistances ellesmêmes, un aspect pour lequel les études de genre se sont avérées essentielles. Nous pouvons par exemple citer les prisons féminines, dans lesquelles les stratégies de survie étaient liées à celles de la résistance quotidienne, comme les deux côtés d'une même pi èce. Comme le démontrera Claudia Cabrero, les actions politiques des premières années de franquisme consistèrent à survivre et à lutter "pour l'hygiène, la nourriture et le renforcement de leurs propres convictions » (Cabrero Blanco, 2006). 
10 Pour rendre visibles ces expériences féminines, qui demeurèrent largement dans l'ombre historiographique, le travail de Giuliana de Febo eut une importance considérable. En 1979, peu après l'instauration de la Constitution en Espagne, elle fut la première à publier sur les résistances féminines (Di Febo, 1979). Comme elle le rappellera elle-même quelques années plus tard dans un état de la question, la tendance était à l'historisation de personnages particulièrement connus, dotant ces biographies d'une perspective largement androcentrique (y compris lorsqu'il s'agissait de biographies de femmes). Lorsque les femmes anonymes apparaissaient, elles étaient «uniquement mentionnées comme "soutien moral" et "aide spirituelle" dans la lutte des hommes", ce qui rendait difficiles les tentatives visant à mettre en avant leurs responsabilités et leurs actions dans ce phénomène. C'était encore chose difficile, car elles-mêmes avaient tendance à minimiser ou nier l'importance de leur intervention qui, dans leur esprit, s'expliquait par un modèle de soins et non par un engagement social et politique, même lorsqu'elles partageaient les postulats de la lutte (Di Febo, 1979, 2006 ; voir aussi Yusta, 2000 : 85).

Dans ce travail de reconnaissance et de revendication des expériences féminines, nous pouvons notamment citer les travaux pionniers de Mercedes Yusta dans l'étude de la résistance maquisarde. Ses recherches firent la lumière sur l'impossibilité de l'existence de tels réseaux de guérilla sans l'implication des femmes. Ainsi, dès ses premières publications, sa volonté d'élargir la notion de résistance au-delà de la simple lutte armée et d'y intégrer la notion de genre aura porté des fruits intéressants (Yusta, 1999, 2003, 2000, 2005). Comme elle l'expose dans l'un de ses derniers livres coordonnés, Heterodoxas, guerrilleras y ciudadanas:

dans leur prétendu apolitisme, l'engagement de ces femmes revêt sans nul doute une signification politique, notamment si nous l'interprétons à la lumière de concepts pensés du point de vue de l'histoire sociale ou de l'anthropologie historique pour donner un sens à des formes d'action propres au monde paysan et qui pourraient s'inscrire dans le cadre des dénommées « révoltes quotidiennes ». Le concept d'« économie morale de la foule », énoncé par E. P. Thompson ou l'« infrapolitique des groupes subalternes » décrite par James $\mathrm{C}$. Scott ouvrent la voie à l'intégration de comportements comme celui de "la Mère » dans une trame collective de solidarités, très souvent féminines, dans laquelle, à partir de valeurs façonnant le fonctionnement des communautés paysannes (telles que la solidarité ou le sens de la justice), il est possible de rejoindre un mouvement comme la résistance antifranquiste, qui confère à ces valeurs un sens politique concret et immédiat (Yusta, 2015).

12 Aujourd'hui, des travaux extraordinaires ont été menés, comme ceux de Claudia Cabrero Blanco, qui utilise la notion de conscience féminine de Temma Kaplan pour étudier la faç on dont les femmes asturiennes ont fait face à la dictature en remplissant leur rôle de mères et de pilier du foyer familial. Dans l'observation des comportements hétérodoxes du point de vue de l'assistance sociale et du travail informel des femmes dans la dictature, les travaux d'Ángela Cenarro, de Sescún Marías, d'Eider de Dios et de Sofía Rodríguez (Cenarro, 2006 ; De Dios, 2014 ; Marías, 2012, 2013 ; Rodríguez, 2010) sont à remarquer. Et concernant l'étude des femmes victimes de rétorsion par la LRP, il convient de mentionner les publications d'Irene Murillo (Murillo, 2013, 2014c, 2016).

En ce sens, il est essentiel de proposer une double analyse tenant compte à la fois des structures et de l'agentivité (ou capacité d'agir), autrement dit, en laissant de côté les approches aprioriques structuralistes (Saz, 1999). Nous retrouvons cette approche dans la notion d'agency développée par les études post-coloniales, ainsi que dans l'histoire de la vie quotidienne allemande (Alltagsgeschichte), où Lüdtke utilise le terme de Eigensinn. Les 
deux idées (agency et Eigensinn) évoqueraient la réappropriation, par le sujet historique, de relations aliénantes, délimitées depuis l'extérieur par des structures et processus dépassant l'acteur, mais qu'il est capable de percevoir et, d'une certaine façon, de contrôler grâce à deux procédés. D'un côté, l'acquisition de ressources fortement liées à la volonté personnelle, l'estime de soi ou le respect de certaines sphères privées considérées comme intouchables. De l'autre, la perspicacité dont il fait preuve, en tant que subalterne, en connaissant sa marge de manœuvre au sein d'un système politique et économique.

Ce prisme s'avère extrêmement intéressant dans notre nécessité éthique et historiographique de ne pas re-victimiser les victimes, mais les observer en mouvement, comme des sujets actifs, des agents sociaux capables d'action et de conscience. En ce sens, Miren Llona reprend l'histoire sociale des années 1970 en revendiquant la place des femmes dans «la libération de l'action humaine de l'entrelacs complexe des relations sociales et des structures politiques et économiques qui l'occultaient" (Llona, 2006). Reprenant une idée développée par Sewell, selon laquelle structure et agentivité ne sont pas opposées, mais, bien au contraire, se requièrent et se sous-entendent mutuellement, nous pouvons affirmer que les structures donnent forme aux pratiques des personnes, tout comme les pratiques des personnes constituent, reproduisent et modifient les structures. En ce sens, il s'agit d'un double processus perméable et changeant, dans lequel

les pensées, motifs et intentions des agents historiques sont façonnés par les cultures et les institutions sociales dans lesquelles ils sont nés, par la façon dont ces cultures et institutions sont reproduites par les structures définies et les actions contraintes de ces agents, mais également, dans certaines circonstances, par la façon dont les agents peuvent (ou doivent) improviser ou innover de certaines façons qui, bien que déterminées structurellement, reconfigurent ces mêmes structures qui les façonnent (Sewell Jr., 1992).

Voyons comment ces modèles théoriques peuvent être appliqués à l'étude des femmes de l'après-guerre espagnole et à leurs stratégies de survie et de résistance.

\section{La lutte contre les mots et les silences}

La mise en marche d'une opération euphémique par la dictature franquiste, notamment à partir de 1939, mais déjà depuis le coup d'État du 18 juillet 1936, nous permet d'affirmer, aux côtés de Godicheau, que lorsque les derniers objectifs militaires furent atteints par l'armée insurgée, le franquisme initia une guerre des mots pour s'approprier la définition de ce qui s'était produit et déposséder le vaincu de ses propres outils d'interprétation et de guérison (Godicheau, 2006). C'est ainsi que fut orchestrée une construction symbolique de la vie et de ce qu'était une vie digne d'être pleurée, comme l'indique Butler, en accord avec la légitimation des machinations sociales et culturelles du pouvoir (Butler, 2010, 2003). Par la distinction entre bonnes et mauvaises vies, cet espace de représentation collective qu'était la mort fut également fragmenté.

17 La gestion des politiques du deuil par le franquisme, déployant une propagande visant à occulter toute version dissidente et tout témoignage de l'Espagne vaincue, impliquait deux opérations. La première consistait en l'hypervisibilité, la commémoration et la sacralisation des morts "dignes d'être pleurées ", c'est-à-dire les morts des vainqueurs perçus comme de bons Espagnols et comme des martyrs, qui demeurèrent dans l'aprèsguerre comme des "morts gardiens», imposant une mémoire hégémonique qui 
prétendait être l'unique voix et l'unique version de la guerre. Finalement, pour reprendre les mots de Zira Box :

si de nombreux morts nés à l'aube de la catastrophe de 1914 furent un ingrédient essentiel de la sacralisation politique à caractère nationaliste menée par les fascistes, la construction symbolique du franquisme repose également sur de nombreux éléments qui avaient pour référence manifeste la guerre dont le régime avait surgi, notamment tout ce qui avait trait à la rhétorique et au rituel entourant les morts (Box, 2010).

La seconde opération consistait en l'ignorance des morts issues de vies de vaincus, les morts républicaines, symboliquement perçues comme des « vies indignes d'être pleurées » et, par conséquent, privées de toute commémoration, de même qu'en l'occultation de la souffrance de ceux qui leur survivaient, qui se voyaient privés du droit individuel de porter le deuil. Ces morts étaient définis comme de "mauvaises vies», ennemis de la nation et responsables de toute la tragédie nationale. Ainsi était écartée toute volonté de " reconnaître, comprendre, aborder et soigner les blessures " d'une société malade et brisée par la souffrance sociale infligée par la guerre et ses agents humains, et par les multiples violences perpétrées et imprégnées dans la vie quotidienne (Collectif, 2009).

L'incapacité des familles des défunts du côté de la République d'offrir à leurs disparus un enterrement physique ou une quelconque transition symbolique, qui aurait permis aux vivants d'aller de l'avant et d'accepter l'absence au quotidien, laissa ces personnes dépourvues de toute opportunité rituelle de dire adieu et de clore la période de la douleur. Néanmoins, cette usure de la mémoire, appelée par Jordi Ibáñez la memoria usurera (mémoire usurière) (Ibáñez, 2009), ferait face dès ses balbutiements à l'opposition d'un secteur de la population qui reprochait à la dictature les droits qu'elle s'arrogeait quant aux identités complexes de la citoyenneté ou aux mémoires plurielles et hétérodoxes en les censurant. Ainsi, la tentative d'homogénéisation du souvenir et de spoliation de la mémoire individuelle et collective se heurta aux versions dissidentes relatives à l'appréhension de la guerre. La violence exercée sur le souvenir ne suffit pas à faire taire les voix qui s'élevaient, parfois timides, parfois véhémentes, face à la culture de la victoire du franquisme sur un terrain très précis : celui de l'appropriation de la sphère sociale dans laquelle les morts de la guerre étaient pleurées, regrettées et gardées dans les mémoires.

Ainsi, la dépossession à laquelle étaient soumis les vivants, dépouillés de l'éthique du deuil, associa leurs réponses à un domaine non seulement éthique, mais également politique. L'étude de sources documentaires fait état d'une voix polysémique qui nous montre les différentes façons dont ces personnes, victimes du franquisme, subissaient et percevaient les violences symboliques et réelles auxquelles elles étaient soumises, et la fa çon dont elles les dénonçaient, les esquivaient et s'y opposaient. L'une de ces résistances fut principalement menée par des femmes, reposant sur des actions de protestation face à la version de l'histoire qui passerait à la postérité. Ce mouvement contestait le fait qu'il appartenait au régime de décider des corps et des vies qui étaient ou non dignes d'être enterrés et pleurés, et la conversion des personnes républicaines en une entité diffuse, un collectif informe qui ôtait à ses membres la précision historique, les parcours personnels ou les raisons particulières qui les avaient convertis en victimes. Ces voix dissonantes constituèrent ainsi l'un des maillons des différentes tensions existant entre le modèle mis en place par le régime et les réponses d'une population exclue du modèle de nation qui, en désaccord avec celui-ci, exigeait le droit d'avoir des droits, d'exercer la mémoire et de 
ne pas voir ses morts exclus de la sphère publique du deuil. Ainsi, il était demandé que soit reconnue aux survivants la souffrance sociale découlant de leurs morts.

La façon dont le camp vainqueur de la Guerre, les putschistes et leurs agents, traitèrent leurs « ennemis » républicains fut une histoire grotesque qui dura plus de mille jours de guerre et dériva ensuite sur des conseils de guerre militaires avec peine de mort. Ainsi, souvenons-nous des exécutions de républicains qui consistaient en des sacas nocturnes, durant lesquelles les personnes étaient emmenées dans des camions pour être exécutées à l'aube et abandonnées dans des fosses communes, sans que ne soit indiqué aux familles l'endroit où gisaient ces personnes, ou du moins les corps de ceux qui autrefois avaient été des personnes. Parmi les témoignages des familles, les précisions sur l'illégalité du processus sont fréquentes, décrivant comment les arrestations étaient menées au moyen des appels d'autorités, parfois réalisés par des personnes de confiance, pour être conduites devant d'autres autorités, généralement de grade supérieur, dans des communes voisines (Carrizosa Isaza, 2011).

En ce sens, certains reprochaient au nouvel État ses origines violentes ou l'illégalité du processus par lequel il avait remporté la guerre. Ils dénonçaient ainsi la violence systématique, légitimée et orchestrée d'en haut, menée par des autorités spécifiques, des agents connus à l'échelle locale. Ces agents ou perpétrateurs n'étaient autres que des habitants de leurs propres communes, des branches familiales proches, des personnes qui dans un cas comme dans l'autre auraient tiré avantage de leur situation et de leur douleur. Constantina Sánchez, résidente de la petite commune de Malón (Tarazona), faisant partie depuis le coup d'État du territoire dominé par l'armée insurgée, décrivait comment :

le 14 janvier 1937, vers six heures du soir, son mari rentrait du travail au champ et, lorsqu'il arriva chez lui accompagné de la déclarante, Eusebio Magaña, le frère de Pedro [son époux], les attendait pour lui dire qu'il devait se rendre à Tarazona immédiatement pour se présenter au quartier général de la Guardia Civil en compagnie de Félix Ruiz et d'autres personnes; comment, obéissant comme toujours aux ordres des autorités, il prépara les chevaux, alla chercher Félix et se rendit avec lui à Tarazona, par le train de septheures, après avoir mangé un morceau de pain, s'en allant en croyant rejoindre les milices, car ils avaient l'intention de s'engager pour défendre la sainte cause dirigée par notre généralissime Franco, mais ne revinrent jamais [...] et comment les rumeurs tenues pour vraies le disaient mort, comme bien d'autres partis avec lui avant d'arriver à Saragosse ${ }^{3}$.

Ce témoignage est complété par celui d'Andresa García, veuve du compagnon de Pedro Magaña, Félix Ruiz. Celle-ci déclarait que, le même jour indiqué plus haut, le 14 janvier 1937, son époux avait reçu :

un message du quartier général de la Guardia Civil de Tarazona lui demandant de se présenter là-bas à 7 heures et demie, où il arriva une heure avant en compagnie de Pedro Magaña. Comme ils ne revinrent pas, le père de Pedro Magaña, Manuel Magaña, partit à Tarazona et, au quartier général, on lui indiqua que seul le garde Quílez pourrait lui répondre. Manuel se rendit chez le garde Quílez qui lui dit qu'ils étaient partis la nuit même pour Saragosse. La rumeur était qu'ils avaient été fusillés au terminus de Magallón ${ }^{4}$.

24 Comme dans le cas précédent, dans lequel Pedro Magaña et Félix Ruiz, entre autres habitants de Malón, «ne sont jamais revenus ", nous pouvons constater que, dans la majorité des témoignages, il est fait allusion aux heures, aux jours et aux mois auxquels la tragédie s'est produite comme une période vécue dans une incertitude angoissante par la 
famille. Les témoignages de la connaissance empoisonnée, comme nous pouvons le voir, ne sont pas restés, du moins pas toujours, dans le domaine privé comme nous l'a habitués à penser l'historiographie. Ces revendications populaires, contestataires de l'histoire officielle, se retrouvent par centaines dans les déclarations et les documents des dossiers de responsabilité civile et politique, détaillant les irruptions et les sacas matinales partout en Aragon et dévoilant les euphémismes du nouvel État.

Eugenia Torralba décrivit point par point le processus de capture, la mobilisation d'autres villages voisins et les délais dans lesquels les détenus étaient tués. Elle déclara que son époux « Francisco Salcedo fut arrêté le 20 août 1936, conduit à Ayerbe [Huesca] où il resta trois jours et, le 24, dans la nuit du 23 au 24, fut emmené et, selon les informations, aurait été fusillé à proximité de Quinzano, où il fut enterré, tout cela durant le mois d'août de l'année passée $»^{5}$. Une autre veuve de Malón, Crescencia Sánchez, âgée de 65 ans, racontait comment :

le 10 septembre, son mari se reposait avec la déclarante lorsque, vers cinq heures du matin, plusieurs hommes frappèrent à la porte, déclarant que la Guardia Civil attendait Jesús Calavia sur la place du village, suite à quoi son mari se leva hâtivement, obéissant aux ordres, et partit avec eux, laissant la déclarante inconsciente; celle-ci apprit par la suite que Jesús et un voisin du village, Emiliano Royo, avaient été conduits à la place où les attendait une voiture de la Guardia Civil qui les emmena, et le jour suivant, la nouvelle fut rendue publique à Malón que tous deux avaient été retrouvés morts à la porte du cimetière de Vierlas, ce que la déclarante ne vit pas elle-même, mais dont il pourra certainement être témoigné dans ledit village ${ }^{6}$.

María Joven Hernández déclara face aux autorités ce qui était déjà de notoriété publique. Son mari, Juan López, secrétaire de la mairie républicaine du village de Bárboles, trouva la mort de manière extrajudiciaire et sans aucune déclaration lorsque,

le 17 août 1936, vers midi, l'huissier municipal se présenta à lui pour lui dire de se rendre à l'hôtel de ville entre deux et trois heures de l'après-midi, ce qu'il fit et suite à quoi il fut transféré à la prison du village, où il resta jusqu'à deux heures du matin le jour suivant, heure à laquelle les phalangistes qui l'avaient arrêté l'emmenèrent et, selon des données fournies officieusement par une personne digne de confiance, l'auraient transféré à Saragosse et l'aurait fusillé sans aucune déclaration, de même que d'autres personnes, et enterré au cimetière catholique de Torrero ${ }^{7}$.

Virgilia Naval, dont le mari José Salavera était membre du comité de direction de l'UGT et le président de la Filial Agraria du village de Belchite, déclara dans son témoignage que Salavera, considéré comme « disparu », " fut appelé par le maire au début de la guerre et, lorsqu'il se présenta, il fut placé en prison, après quoi il aurait été retrouvé mort dans un ravin du village de María avec d'autres habitants de Belchite ». Jesús Labasa, patron de bistrot à Alfajarín, se rendit dans la rue pour empêcher le putsch lorsque les nouvelles du soulèvement arrivèrent à ce village de Saragosse. Lorsque les insurgés prirent la commune, ils allèrent le chercher chez lui, l'emprisonnèrent dans la ville et firent écrire par toutes les sources qu'il était porté " disparu », guillemets inclus. Dans sa déclaration, l'épouse nia le témoignage officiel et affirma savoir de sources sûres que son mari était mort le 8 septembre 1936 dans le quartier de Movera, où son cadavre aurait été retrouvé8. Dans d'autres cas, les veuves contestèrent les informations officielles en pointant du doigt leurs incohérences et en confrontant les autorités avec une version des faits qui mettait en lumière le caractère vexatoire de données incorrectes et insultantes, car il s'agissait de personnes de chair et d'os à la disparition desquels les familles tentaient de faire face. 
L'épouse de Nicolás Esteban, métreur à Mas de las Matas (Teruel), confronta la version officielle du régime, dont

l'élément principal est que celle-ci affirme que mon mari, Nicolás Esteban Gasión, « disparut le 28 mai 1938, et serait passé à la zone rouge et mort à Morella sur le front de Castellón ». Face à cette déclaration, il ne fait aucun doute qu'il ne s'agit pas de Nicolás Esteban Gasión, car celui-ci, suite à la libération du village de Mas de las Matas, s'y rendit après avoir été libéré par les autorités, car aucune charge ne pesait contre lui et, après quinze jours passés à Mas de las Matas, il fut appelé au bureau de la Phalange le 28 mai 1938 et, sans aucune autre nouvelle (officielle), fut enterré avec d'autres résidents au cimetière dudit village. Cela est du domaine public à Mas de la Matas, de même que le moment, le lieu et la raison de sa mort. Et tout le monde sait qu'il n'est pas passé à la zone rouge et qu'il n'est pas mort au front, ni dans le village de Morella9.

Connaître ces faits supposait d'être en possession d'un passé qui entrait dans le présent comme une "connaissance empoisonnée" (Das, 2006). Avec cette connaissance empoissonnée et ce témoignage, il était également question de laver les noms des défunts et de les intégrer au paysage de l'après-guerre. Ce savoir, ce témoignage de ce qui fut et demeure dans sa réactualisation, est ce que Martha Nussbaum appelait la « connaissance par la souffrance ", faisant allusion à la façon dont la souffrance, l'émotion incarnée de la souffrance personnelle, est toujours associée à la connaissance, au récit de ce qui a été vécu et à la résistance à l'indifférence, à l'injustice et à l'oubli (Nussbaum, 1986). Fiona C. Ross, qui a étudié les récits offerts par la population sud-africaine dans le cadre de la Commission de vérité et de réconciliation en Afrique du Sud à partir de 1994, considère que «la mémoire et la connaissance sont intimement liées » et que, par conséquent, la mémoire des victimes ne peut être séparée de ce qui leur a infligé la souffrance (Ross, 2001). En Espagne, José María Ruíz Vargas, dans son étude des événements traumatiques tels que la guerre civile d'Espagne ou, plus récemment, les attentats du métro de Madrid (11-M), intègre un nouvel élément: le lien entre émotion et mémoire, par lequel la première fonctionnerait sans aucun doute comme facteur d'accroissement de la seconde. Ainsi, « bien que les relations entre les deux [émotion et mémoire] soient perçues comme d'une extraordinaire complexité, il semblerait qu'il n'existe aucun ingrédient plus efficace que les sentiments, les affects et les émotions de manière générale pour que les expériences humaines restent gravées au fer rouge dans notre mémoire » (Ruiz Vargas, 2015 ; Virgili, 2006).

La perspective proposée par le témoignage des victimes était d'ordre éthico-politique dans le sens où ceux qui réagissaient et s'opposaient à la mémoire usurière considéraient que l'événement n'avait pas eu de dimension arbitraire et fortuite face à laquelle il n'y avait aucun responsable, ni de dimension providentielle ou nécessaire, comme l'avançait la dictature elle-même, mais que le malheur de la guerre avait été un choix politique et, par conséquent, aurait pu être évité. Les témoignages de personnes aragonaises que nous étudions ci-après reflètent leur "souffrance sociale " comme un affront et le souvenir officiel comme une moquerie. Cet élan de dignité et de mémoire, que nous pouvons observer dans la plupart des témoignages, est particulièrement présent dans celui de Soledad Fuertes, veuve du fonctionnaire de la Confederación Hidrográfica del Ebro, Francisco Oliva. Celle-ci a mis trois ans à accepter l'exécution de son époux et c'est seulement à ce moment-là qu'elle écrivit afin d'« évacuer le processus de disculpation » :

Au départ, je ne pensais pas écrire ce récit, à cause de toute la douleur et la peine que représente pour moi tout ce qui se rattache à la personne que j'ai perdue, la personne que j'aimais le plus au monde, dont la bonté, l'honneur et l'intégrité ont 
toujours été loués par tous ceux qui le connaissaient et le fréquentaient. Puis, en y repensant, j'ai décidé de le faire, non pas pour moi, mais pour nos enfants, qui ont parfaitement le droit de voir la mémoire de leur père rétablie et son nom resplendir, pour qu'ils puissent porter fièrement le leur, la tête haute [...]. Ce que faisait mon époux, et qui lui valait la sympathie générale, était de répondre à toutes les demandes qui lui étaient adressées, offrant ses services professionnels à tous ceux qui en avaient besoin, sans se demander si ses clients avaient ou non des moyens et s'ils pourraient s'acquitter de ses honoraires; bien au contraire, il s'occupait avec autant de soin, si ce n'est plus, des personnes dans le besoin qui ne pouvaient le payer ${ }^{10}$.

31 Après quatre pages passées à décrire et défendre le caractère et les agissements de Francisco Oliva, Soledad Fuertes termine sur une phrase sans équivoque: "Je ne m'étendrai pas davantage afin de ne pas abuser de l'attention judiciaire et, car la douleur et la peine causées par le souvenir de mon époux et de son triste sort sont trop grandes, mais je dois faire tout cela, et plus encore, si cela est nécessaire afin de réhabiliter sa mémoire ».

Eloisa Adán, veuve de l'agriculteur Antonio Adán, contesta devant le tribunal les charges qui pesaient contre son époux et dénonça le manque de compassion du régime, qui jouait avec les vies des individus comme s'il était Dieu, alors que seul le Seigneur « est maître de nous tous ". La veuve écrivit :

Comment mon pauvre époux aurait-il pu être l'auteur de tous les désordres commis dans la commune, qui lui sont attribués dans ledit rapport, s'il est reconnu, d'un autre côté, qu'il est mort peu de temps après le début du mouvement (il est tombé malade le 20 septembre 1936 et mort sans avoir quitté le lit le 19 octobre 1936)? Comment un homme qui n'a vécu que 63 jours durant la période révolutionnaire aurait-il pu, durant cette période et même après (ledit rapport laissant entendre qu'il serait coupable de tous les faits commis dans la commune), être l'auteur de ces faits? Monsieur, ayez je vous prie un peu de charité pour les défunts et un peu de respect pour tous ceux qui, s'il avait commis un pêché, purgeraient actuellement le châtiment divin du Seigneur, qui est en définitive notre seul maître et seigneur à tous, et qui seul peut punir le fait d'avoir une idée qui n'aura jamais nui à personne et que nul ne peut affirmer ni encore moins prouver dans cette vie ${ }^{11}$.

33 Face à une mémoire usurière considérant que l'organe endommagé suite au conflit était l'organe politique de la nation et que le collectif républicain était l'auteur matériel de tous les dommages qu'avaient subis la nation et les individus, se dressaient les contestations de ceux qui avaient connu l'autre version de cette hégémonie du souvenir. Face à la dépréciation des expériences individuelles et à l'expulsion de la douleur du domaine public, les récits de ces personnes n'étaient pas seulement émotionnels et domestiques, bien que basés sur leurs propres expériences et sentiments, mais ils intégraient également, par le biais de l'émotion, l'arme politique qu'était la connaissance de ce qui s'était produit, le fait de savoir quelque chose que l'État niait.

\section{Conclusions}

Les stratégies de résistance des femmes avaient pour objectif principal de minimiser, dans leur quotidien, l'appropriation par le pouvoir de leurs vies individuelles et de leurs relations sociales. Tant le projet classiste et sexiste de la dictature que les différents modes de dépossession matérielle et immatérielle de la population vaincue par le régime étaient sujets à la contestation. Dans le cadre de la récupération de la dignité matérielle, nous retrouvons toutes sortes d'actions visant à atteindre une certaine autonomie. Dans 
le domaine de la dépossession immatérielle, la lutte en vue d'une réassurance éthique fut durement menée. L'écriture, par exemple, constitua un mécanisme clé qui permit aux femmes de revendiquer leur voix et leur propre vision des événements, laissant à l'histoire de nombreux récits alliant au témoignage la connaissance et à la connaissance la dénonciation. En ce sens, en tant que survivantes de la Guerre et représentantes de la mémoire, le travail de plusieurs femmes de l'après-guerre peut sans nul doute être consid éré comme pionnier de la dénommée mémoire historique.

Nous soutenons que la perspective proposée par le témoignage des veuves, victimes de la violence symbolique et matérielle, était d'ordre éthico-politique en ce sens où répondre à la mémoire usurière du régime supposait d'affirmer ouvertement que l'événement de la guerre n'avait pas eu de dimension arbitraire et fortuite face à laquelle il n'y avait aucun responsable, ni de dimension providentielle ou nécessaire, comme l'avançait la dictature elle-même, mais que ce malheur avait été un choix politique et, par conséquent, aurait pu être évité. Selon nous, le personnage d'Antigone, transféré à l'après-guerre espagnole, va bien plus loin. Le fait de réclamer le deuil familial pour ces vies perçues par le pouvoir comme des non-vies, comme des vies indignes d'être pleurées ou enterrées, comme des croque-mitaines « rouges » tenus responsables de la guerre, représente un acte éthique et politique d'affrontement au régime. Cela implique de lutter pour résister à l'appropriation par le pouvoir de la vie d'autrui, de son histoire et de sa mémoire, ce que l'on pourrait qualifier de deuil militant ou, à l'instar de certaines écrivaines telles que Judith Butler, Douglas Crimp, ou Athena Athanasiou, de « deuil activiste » (Athanasiou, 2013 ; Butler, 2003, 2010 ; Crimp, 1989). Cela implique également de dénoncer la violence de l'État, qui a outrepassé les limites, empiétant sur des territoires qui devraient être dominés par d'autres principes comme, au-delà des lois politiques, les lois de l'éthique.

Des concepts tels que la résistance civile et les rébellions quotidiennes aident à mettre en évidence et à doter d'une signification politique les actions de groupes traditionnellement peu visibles dans l'action collective, comme ici celui des femmes. Ainsi, à la lumière de ces contributions, nous pouvons donner de l'intelligibilité et une signification politique à toute une série de comportements et d'actions adoptés par des femmes et qui, au-delà de leur appartenance apparente à la sphère privée et quotidienne, constituent les témoignages d'une sourde opposition à la dictature sous des formes différant des plus connues et visibles.

Les victimes de la Loi négociaient comme elles le pouvaient avec la législation pour tenter d'échapper à la sanction (Cenarro, 2009; Murillo, 2014, 2016). Le moteur d'action de ces femmes émanait de cette autoconscience de l'exclusion et du mépris. C'est de là, de la conscience d'être des vies soumises au contrôle et à la répression, que part leur résistance. En tant que victimes d'une violence spécifique, elles élaborent des stratégies de défenses spécifiques (Fulchirone, 2011; Suárez Navaz \& Hernández, 2011). Dans cette autoreprésentation en tant que victimes, elles parviennent à concevoir un discours propre qui constitue, sous de nombreux aspects, une résistance au franquisme. Dans leurs revendications du droit à un traitement juste, elles dévoilent les mauvais traitements du régime; dans leur formulation de l'illégitimité des processus de confiscation de biens, elles dénoncent l'illégitimité du régime; dans la mise en marche de stratégies de solidarité envers d'autres victimes de représailles, elles rejettent la "table rase» impliquant l'exclusion de tout vaincu, considéré comme anti-Espagne et ennemi de l'État. celui de survivantes. Cette transcendance et ce statut de survivantes (et résistantes) ont 
des répercussions directes sur leurs conditions de vie. En effet, le fait d'associer à l'émotion d'indignation morale la connaissance et la palliation de cette dernière consistait à faire un pas en avant dans la prise de contact avec ceux qui étaient considérés comme à l'origine de ce grief ou de cette exclusion, leur demandant la mise en place de mesures visant à pallier l'injustice.

Pour cette raison, nous devons aller plus loin dans notre analyse de la violence, en examinant non seulement le contexte dans lequel un monde est détruit, mais également la façon dont celui-ci est reconstruit par les survivants et la façon dont sont perçues les relations et les pratiques de pouvoir, la relation avec l'État et entre les citoyens euxmêmes, lorsque tout doit être rebâti. En ce sens, nous nous lançons le défi de parvenir à écrire comment les communautés et les personnes qui les composent parviennent à faire face à la violence traumatique et au contexte de souffrance sociale et de dévastation qu'un événement comme une guerre civile laisse derrière lui : comment cette violence est perçue, endurée, transcendée et supportée (Das, 2006).

\section{BIBLIOGRAPHIE}

ANDERSON Peter (2013), «¿Amigo o enemigo? La construcción de la verdad franquista sobre el pasado en guerra tras la ocupación», Ó. Rodríguez Barreira (dir.), El Franquismo desde los márgenes: Campesinos, mujeres, delatores, menores..., Lleida : Espai/Temps, 77-91.

ARÓSTEGUI Julio (2012), «Coerción, violencia, exclusión. La dictadura de Franco como sistema represivo», J. Aróstegui (dir.), Franco: la represión como sistema, Barcelone : Flor del Viento ediciones, 19-59.

Athanasiou Athena (2005), «Reflections on the Politics of Mourning: Feminist Ethics and Politics in the Age of Empire», Historein, 5, 40-57.

BARRADO GRACIA Javier (1999, noviembre), «Mujeres y derrota. La represión de la mujer en el Teruel de posguerra (1939)», communication présentée au Tiempo de Silencio. IV Encuentro de investigadores del Franquismo, Valence, Espagne.

Box Zira (2010), Espania an a cero: la construccio-in simbo lica del franquismo, Madrid : Alianza Editorial.

BUTLER Judith (2003), «Violencia, luto y política», Iconos, Revista de Ciencias Sociales, 17, 82-99.

BUTLER Judith (2010), Marcos de guerra. Las vidas lloradas, Barcelone : Paidós.

BUTLER Judith \& ATHANASIOU Athena (2013), Dispossession: The performative in the political, Cambridge : Polity.

CABRERo BlANCo Claudia (2006), Mujeres contra el franquismo (Asturias 1937-1952). Vida cotidiana, represión y resistencia, Oviedo : KRK.

CARRIzosa ISAZA Catalina (2011), «El trabajo de memoria como vehículo de empoderamiento político: la experiencia del Salón del Nunca Más», Boletín de Antropología, 25(42), 36-56. 
CENARro Ángela (2006), La sonrisa de Falange. Auxilio Social en la Guerra Civil y en la posguerra, Barcelone : Crítica.

CENARRo Ángela (2009), Los niños del Auxilio Social, Barcelone : Espasa.

CENARRo Ángela (2016), «Entre el maternalismo y el pronatalismo: el Seguro de Maternidad en los orígenes de la dictadura de Franco (1938-1942)», Ayer, 102(2), 47-70.

collectif (2009), Tejidos que lleva el alma. Memoria de las mujeres mayas sobrevivientes de violación sexual durante el conflicto armado, Guatemala: Equipo de Estudios Comunitarios y Acción Psicosocial y Unión Nacional de Mujeres Guatemaltecas.

CRIMP Douglas (1989), «Mourning and militancy», October, 51, 3-18.

DAS Veena (2006), Life and Words. Violence and Descent into the Ordinary, Oxford : Oxford University Press.

DAs Veena, Kleinman Arthur, Lock Margaret, RAmPhele Mamphela \& Reynolds Pamela (2001), Remaking a World. Violence, Social Suffering and Recovery, University of California Press.

DAviEs Douglas J. (2000), «Rober Hertz: The social triumph over death», Mortality, 5(1), 97-102.

DE Dios Eider (2014), «Domesticidad y familia: ambigüedad y contradicción en los modelos de feminidad del franquismo», Feminismo/s, 23, 23-46.

Del ARCo BlANCo Miguel Ángel (2009), «El secreto del consenso en el régimen franquista: cultura de la victoria, represión y hambre», Ayer, 76(4), 245-268.

Del Arco Blanco Miguel Ángel, Fuertes Carlos, Hernández Burgos Claudio \& Marco Jorge (2013), No solo miedo. Actitudes políticas y opinión popular bajo la dictadura franquista (1936-1977), Granada : Comares Historia.

Di Feво Giuliana (1979), Resistencia y movimiento de mujeres en España, 1936-1976, Barcelone : Icaria.

Di FEBo Giuliana (2006), «Resistencias femeninas al franquismo. Para un estado de la cuestión», Cuadernos de Historia Contemporánea, 28, 153-168.

FulChiRone Amandine et al. (2011), Yo soy voz de la memoria y cuerpo de la libertad, Ciudad de Guatemala : Colectiva Actoras del Cambio.

GODICHEAU François (2006), «Guerra civil, guerra incivil: la pacificación por el nombre», J. Aróstegui et F. Godicheau (dir), Guerra civil. Mito y memoria, Madrid : Marcial Pons/Casa de Velázquez, 137-166.

IBÁÑEZ FANÉs Jordi (2009), Antígona y el duelo. Una reflexión moral sobre la memoria histórica, Barcelone : Ensayo Tusquets.

LAGARTA Estefanía, MORENO Nacho \& MURILlo Irene (2014), «Las víctimas de la represión económica en Aragón», J. Casanova \& Á. Cenarro (dir.), Pagar las culpas. La represión económica en Aragón (1936-1945), Barcelone : Crítica, 41-96.

LLONA Miren (2006), «Género e identidad de clase. La construcción de la clase obrera vizcaína durante el primer tercio del siglo XX», Historia Social, 54, 95-112.

LÜDTKE Alf (1995), «De los héroes de la resistencia a los coautores. “Alltagsgeschichte” en Alemania», Ayer, 19, 49-70.

MARíAs Sescún (2013a), «El empleo femenino a los dos lados del margen: la Sección Femenina y el trabajo de la mujer», Ó. Rodríguez Barreira (dir.), El Franquismo desde los márgenes: Campesinos, mujeres, delatores, menores..., Lleida : Espai/Temps, 147-163. 
MARÍAS Sescún (2013b), La Sección Femenina y el control de las trabajadoras. Zaragoza, 1939-1975 (tesis doctoral).

MIR Conxita, CORRETGÉ Fabià, FARRÉ Judit \& SAGUÉS Joan (1997), Repressió econòmica i franquisme: $l$ 'actuació del Tribunal de Responsabilitats Polítiques a la provincia de Lleida, Barcelone : Publicacions de l'Abadia de Monserrat.

MIR Conxita (2004), «La represión sobre las mujeres en la posguerra española», Cuadernos Republicanos, 54, 205-227.

MOLINERo Carme (1998), «Mujer, franquismo, fascismo. La clausura forzada en un mundo pequeño», Historia Social, 30, 97-117.

MURILLO ACED Irene (2013), En defensa de mi hogar y mi pan. Estrategias femeninas de resistencia civil y cotidiana en la Zaragoza de posguerra, 1936-1945, Saragosse : Prensas Universitarias de Zaragoza.

MURILLO ACED Irene (2014a), «“A Vuestra Excelencia con el mayor respeto y subordinación”. La negociación de la Ley desde abajo», J. Casanova \& Á. Cenarro (dir.), Pagar las culpas. La represión económica en Aragón (1936-1945), Barcelone : Crítica, 203-226.

MURILLO ACED Irene (2014b), «"Ni moral, ni justo, ni legal, ni humano”. Resistencias civiles al franquismo», J. Casanova \& Á. Cenarro (dir.), Pagar las culpas. La represión económica en Aragón (1936-1945), Barcelone : Crítica, 227-253.

MURILLO ACED Irene (2014c), «Dignidad, supervivencia y luto. Agencias y resistencias de mujeres aragonesas de guerra y posguerra», M. Yusta \& I. Peiró (dir.), Heterodoxas, guerrilleras y ciudadanas. Formas de resistencia femenina en la España moderna y contemporánea, Saragosse : Institución Fernando el Católico, 149-174.

MURILLO ACED Irene (2016), Exigiendo el derecho a tener derechos: ciudadanía y género como prácticas de negociación y resistencia: el caso de Aragón, 1936-1945, <http://zaguan.unizar.es/record/47865>.

NASH Mary (1996), «Pronatalismo y maternidad en la España franquista», G. Bock \& P. Thane (dir.), Maternidad y políticas de género: las mujer en los estados de bienestar europeos, 1880-1950, Madrid : Cátedra, 279-308.

NASH Mary (2013), Represión, resistencias, memoria: las mujeres bajo la dictadura franquista evidencia la centralidad del género en el desarrollo del franquismo, Granada : Editorial Comares.

NusSBAum Martha (1986), The Fragility of Goodness: Luck and Ethics in Greek Tragedy and Philosophy, Londres : Cambridge University Press.

RoDRíGUEZ Sofía (2010), «La Sección Femenina, la imagen del poder y el discurso de la diferencia», Feminismo/s, 16, 233-257.

RoDRíGUEz Sofía (2013), «Entre líneas. Estudiar a las mujeres desde los márgenes al aparato», Ó. Rodríguez Barreira (dir.), El Franquismo desde los márgenes: Campesinos, mujeres, delatores, menores..., Lleida : Espai/Temps, 131-145.

Ross Fiona C. (2001), «Speech and Silence. Women's Testimony in the First Five Weeks of Public Hearings of the South African Truth and Reconciliation Commission», V. Das, A. Kleinman, M. Lock, M. Ramphele \& P. Reynolds (dir.), Remaking a World. Violence, Social Suffering and Recovery, University of California Press, 250-279.

RUIZ VARGAS José María (2006), «Trauma y memoria de la Guerra Civil y la dictadura franquista», Hispania Nova. Revista de Historia Contemporánea, 6, <http://hispanianova.rediris.es/6/ dossier/6d012.pdf>. 
SAZ Ismael (1999), «Entre la hostilidad y el consentimiento. Valencia en la posguerra», I. Saz \& A. Gómez Roda (dir.), El franquismo en Valencia. Formas de vida y actitudes sociales en la posguerra, Valence : Episteme.

SEWELL JR. William H. (1992), «A Theory of Structure: Duality, Agency, and Transformation», American Journal of Sociology, 98(1), 1-29.

SuÁReZ NAVAZ Liliana \& HERnÁNDEZ Rosalva Aída (2011), Descolonizando el feminismo. Teorías y prácticas desde los márgenes, Valence : Cátedra.

TAMARIT Sumalla Josep María (2005), «Derecho penal y delincuencia en la legislación de posguerra», C. Mir, C. Agustí \& J. Gelonch (dir.), Pobreza, marginación, delincuencia y políticas sociales bajo el franquismo, Lleida : Servei de Publicacions de la Universitat de Lleida, 53-55.

VALIENTE Celia (2003), «Las políticas para las mujeres trabajadoras durante el franquismo», G. Nielfa Cristóbal (dir.), Mujeres y hombres en la España franquista: Sociedad, economía, política, cultura, Madrid : Universidad Complutense, 145-180.

VIRGILI Fabrice (2006), «Víctimas, culpables y silenciosas: memorias de las mujeres rapadas en la Francia de la posguerra», J. Aróstegui \& F. Godicheau (dir.), Guerra civil. Mito y memoria, Madrid : Marcial Pons/Casa de Velázquez, 361-372.

YUSTA Mercedes (1999), La guerra de los vencidos. El maquis en el Maestrazgo turolense, 1940-1950, Saragosse : Institución Fernando el Católico.

YUSTA Mercedes (2000), «Sujetos femeninos en espacios “masculinos": la resistencia al franquismo de las mujeres aragonesas (1940-1950)», El siglo Xx: balance y perspectivas, Valence : Fundación Cañada Blanch, 85.

YUSTA Mercedes (2003), Guerrilla y resistencia campesina: la resistencia armada contra el franquismo en Aragón (1939-1952), Saragosse : Prensas Universitarias de Zaragoza.

YUSTA Mercedes (2005), «Las mujeres en la resistencia antifranquista: un estado de la cuestión», Arenal, 12(1), 5-34.

YUSTA Mercedes (2015), «Con armas frente a Franco, Mujeres guerrilleras en la España de posguerra», M. Yusta et I. Peiró (dir.), Heterodoxas, guerrilleras y ciudadanas. Formas de resistencia femenina en la España moderna y contemporánea, Saragosse : Institución Fernando el Católico.

\section{NOTES}

1. Cet article a été rédigé dans le cadre du projet d'investigation «Discursos, identidades y políticas de género en la derecha española y la Dictadura de Franco, 1875-1975» (HAR2015-63624P).

2. Loi sur les responsabilités politiques du 9 février 1939.

3. AHPZ, FRP, Expediente 5741/2.

4. AHPZ, FRP, Expediente 5741/8.

5. AHPZ, FRP, Expediente 5962/5.

6. AHPZ, FRP, Expediente 5740/8.

7. AHPZ, FRP, Expediente 5562/8. L'italique a été ajouté par mes soins.

8. AHPZ, FRP, JP, Expediente 7005/20 y AHPZ, FRP, Expediente 5520 /13.

9. AHPT, FRP, Expediente 236/19.

10. AHPZ, FRP, Expediente 5938/13.

11. AHPT, FRP, Expediente 236/18. 


\section{RÉSUMÉS}

Le franquisme visait à la dépossession matérielle et immatérielle de la population vaincue. La Loi sur les responsabilités politiques est le paradigme même de cette double volonté répressive. $\mathrm{L}$ 'objectif de cet article est d'analyser les résistances féminines à la privatisation de la souffrance sociale de la population vaincue. Face à la dépréciation des expériences individuelles et à l'expulsion de la douleur du domaine public, les récits de ces personnes n'étaient pas seulement émotionnels et domestiques, bien que basés sur leurs propres expériences et sentiments, mais ils intégraient également, par le biais de l'émotion, l'arme politique qu'était la connaissance de ce qui s'était produit, le fait de savoir quelque chose que l'État niait.

El propósito de la dictadura franquista fue expropiar a la población vencida, material e inmaterialmente. La Ley de Responsabilidades Políticas es el paradigma mismo de esta doble ambición represora. Este artículo se ocupa de las resistencias femeninas a la privatización del sufrimiento social como fenómeno exclusivo del bando vencedor. Ante el desprecio de las experiencias individuales y la expulsión del dolor del campo público, los relatos de centenares de mujeres no eran únicamente emocionales y domésticos, sino que mediante la emoción se incorporaba el arma política del conocimiento de lo ocurrido, de saber algo que el Estado negaba y de señalar y condenar al Nuevo Estado por sus orígenes violentos o la ilegalidad del proceso que les había aupado como vencedores de la guerra.

Francoism purpose was to expropriate defeat population both materially and immaterially. 1939's Law of Political Responsibilities is the paradigm of that doubled-face repression. This article delve into women resistance to social suffering privatization' as a victors unique phenomena. In the face of Francoism lack of qualms about individual experiences and due to the expulsion of suffering from public domain, the narratives of hundreds of women took up a strong and critical stance towards the Nuevo Estado. Rather than study their narratives as private stories, we should rather bring their emotions to the fore and research their knowledge as a political weapon against Francoism. In their writings they indict violent origins and deplore dictatorship process of victory as a victory drove through illegality and extreme violence.

\section{INDEX}

Palabras claves : franquismo, sufrimiento social, escritura, agencia, dignidad, viudedad, emoción, memoria, resistencia

Keywords : francoism, social suffering, narrative, agency, dignity, widowhood, emotion, memory, resistance

Mots-clés : franquisme, souffrance sociale, récit, agence, dignité, veuvage, émotion, mémoire, résistance 


\section{AUTEUR}

\section{IRENE MURILLO ACED}

Chercheuse et docteur de l'Université de Saragosse (Espagne).

Auteur de En defensa de mi hogar y mi pan: estrategias femeninas de resistencia civil y cotidiana en la Zaragoza de posguerra, 1936-1945, Universidad de Zaragoza (2013).

Thématiques de recherche : Répression économique et formes de résistances aux dictatures. irenemurilloaced@gmail.com 